\title{
INTERVAL-VALUED FUZZY SUBGROUPS AND RINGS
}

\author{
HeE Won KanG* And Kul HuR**
}

\begin{abstract}
We introduce the concepts of interval-valued fuzzy subgroups [resp. normal subgroups, rings and ideals] and investigate some of it's properties.
\end{abstract}

\section{Introduction}

In 1986, Atanassuv[1] introduced the concept of intuitionistic fuzzy sets as a generalitation of fuzzy sets introduced by Zadeh[13], After then, Banerjee and Basnet[3], and Hur et. al[8, 9] applied it to algebra. Çoker $[5,6]$ studied intuitionistic fuzzy topological spaces.

In 1975, Zadeh[14] suggested the notion of interval-valued fuzzy sets as another generalization of fuzzy sets. After that time, Biswas[4] applied it to group theory, and Gorzalczany[7] suggested a method of inference in approximate reasoning by using interval-valued fuzzy sets. Moreover Montal and Samanta[12] introduced the concept of topology of interval-valued fuzzy sets and investigate some of it's properties. Recently, Hur et. al[10] studies interval-valued fuzzy relations in the sense of a lattice theory. In this paper, we introduce the concept of intervalvalued fuzzy subgroups [resp.normal subgroup, rings and ideals] and investigate some of it's properties.

\section{Preliminaries}

Received July 26, 2010. Accepted November 3, 2010.

** Corresponding author.

2000 Mathematics Subject Classification : 20N25.

Keywords and phrases. t-norm : interval-valued fuzzy subgroup[ring and ideal], interval-valued fuzzy normal subgroup. 
In this section, we list some concepts and results related to intervalvalued fuzzy set theory and needed in next sections.

Let $D(I)$ be the set of all closed subintervals of the unit interval $[0,1]$. The elements of $D(I)$ are generally denoted by capital letters $M, N, \cdots$, and note that $M=\left[M^{L}, M^{U}\right]$, where $M^{L}$ and $M^{U}$ are the lower and the upper end points respectively. Especially, we denoted, $\mathbf{0}=[0,0], \mathbf{1}$ $=[1,1]$, and $\boldsymbol{a}=[a, a]$ for every $a \in(0,1)$, We also note that

(i) $(\forall M, N \in D(I))\left(M=N \Leftrightarrow M^{L}=N^{L}, M^{U}=N^{U}\right)$,

(ii) $(\forall M, N \in D(I))\left(M=N \leq M^{L} \leq N^{L}, M^{U} \leq N^{U}\right)$.

For every $M \in D(I)$, the complement of $M$, denoted by $M^{C}$, is defined by $M^{C}=1-M=\left[1-M^{U}, 1-M^{L}\right](\operatorname{See}[12])$.

Definition 2.1[7,14]. A mapping $A: X \rightarrow D(I)$ is called an intervalvalued fuzzy set(is short, IVFS) in $X$, denoted by $A=\left[A^{L}, A^{U}\right]$, if $A^{L}, A^{L} \in I^{X}$ such that $A^{L} \leq A^{U}$, i.e., $A^{L}(x) \leq A^{U}(x)$ for each $x \in X$, where $A^{L}(x)\left[\operatorname{resp} A^{U}(x)\right]$ is called the lower[resp upper] end point of $x$ to $A$. For any $[a, b] \in D(I)$, the interval-valued fuzzy $A$ in $X$ defined by $A(x)=\left[A^{L}(x), A^{U}(x)\right]=[a, b]$ for each $x \in X$ is denoted by $\left.\widetilde{[a, b}\right]$ and if $a=b$, then the IVFS $[\widetilde{a, b}]$ is denoted by simply $\widetilde{a}$. In particular, $\widetilde{0}$ and $\widetilde{1}$ denote the interval-valued fuzzy empty set and the interval-valued fuzzy whole set in $X$, respectively.

We will denote the set of all IVFSs in $X$ as $D(I)^{X}$.It is clear that set $A=[A, A] \in D(I)^{X}$ for each $A \in I^{X}$.

For sets $X, Y$ and $Z, f=\left(f_{1}, f_{2}\right): X \rightarrow Y \times Z$ is called a complex mapping if $f_{1}: X \rightarrow Y$ and $f_{2}: Y \rightarrow Z$ are mappings.

Definition 2.1' ${ }^{\prime}[1,9]$. Let $X$ be a set. A complex mapping $A=$ $\left(\mu_{A}, \nu_{A}\right): X \rightarrow I \times I$ is called a intuitionistic fuzzy set(in short, IFS) in $X$ if $\mu_{A}(x)+\nu_{A}(x) \leq 1$ for each $x \in X$, where the mappings $\mu_{A}: X \rightarrow I$ and $\nu_{A}: X \rightarrow I$ denote the degree of membership(namely $\left.\mu_{A}(x)\right)$ and the degree of nonmembership(namely $\nu_{A}(x)$ ) of each $x \in X$ to $A$, respectively. in particular, $0_{\sim}$ and $1_{\sim}$ denote the intuitionistic fuzzy empty set and intuitionistic fuzzy whole set in $X$ defined by $0_{\sim}(x)=(0,1)$ and $1 \sim(x)=(1,0)$ for each $x \in X$, respectively.

We will denoted the set of all the $\operatorname{IFSs}$ in $X$ as $\operatorname{IFS}(X)$. 
Result 2.A[2, Lemma 1]. We define two mappings $f: D(I)^{X} \rightarrow \operatorname{IFS}(\mathrm{X})$ and $g: \operatorname{IFS}(\mathrm{X}) \rightarrow \mathrm{D}(\mathrm{I})^{\mathrm{X}}$ as follows, respectively:

(i) $f(A)=\left(A^{L}, 1-A^{U}\right), \forall A \in D(I)^{X}$,

(ii) $g(B)=\left[\mu_{B}, 1-\nu_{B}\right], \forall B \in \operatorname{IFS}(\mathrm{X})$.

In this case, we write as $f(A)=A_{*}$ and $g(B)=B^{*}$, respectively. Then

(a) $g \circ f=1_{D(I)^{X}}$, i.e., $g(f(A))=A, \forall A \in D(I)^{X}$.

(a) $f \circ g=1_{\mathrm{IFS}(\mathrm{X})}$, i.e., $f(g(B))=B, \forall B \in \operatorname{IFS}(X)$.

Definition 2.2[7]. An IVFS A is called an interval-valued fuzzy point(in short, IVFP) in $X$ with the support $x \in X$ and the value $[a, b] \in D(I)$ with $b>0$, denoted by $A=x_{[a, b]}$, if for each $y \in X$

$$
A(y)=\left\{\begin{array}{c}
{[a, b] \text { if } y=x} \\
0 \text { otherwise }
\end{array}\right.
$$

In particular, if $b=a$, then $x_{[a, b]}$ is denoted by $x_{\boldsymbol{a}}$.

We will denote the set of all IVFPs in $X$ as $\operatorname{IVF}_{\mathrm{P}}(\mathrm{X})$.

Definition 2.3 [7]. Let $A, B \in D(I)^{X}$ and let $\left\{A_{\alpha}\right\}_{\alpha \in \Gamma} \subset D(I)^{X}$. Then:

(i) $A \subset B$ iff $A^{L} \leq B^{L}$ and $A^{U} \leq B^{U}$.

(ii) $A=B$ iff $A \subset B$ and $B \subset A$.

(iii) $A^{C}=\left[1-A^{U}, 1-A^{L}\right]$.

(iv) $A \cup B=\left[A^{L} \vee B^{L}, A^{U} \vee B^{U}\right]$.

$(\mathrm{iv})^{\prime} \bigcup_{\alpha \in \Gamma} A_{\alpha}=\left[\bigvee_{\alpha \in \Gamma} A_{\alpha}^{L}, \bigvee_{\alpha \in \Gamma} A_{\alpha}^{U}\right]$.

(v) $A \cap B=\left[A^{L} \wedge B^{L}, A^{U} \wedge B^{U}\right]$.

$(\mathrm{v})^{\prime} \bigcap_{\alpha \in \Gamma} A_{\alpha}=\left[\bigwedge_{\alpha \in \Gamma} A_{\alpha}^{L}, \bigwedge_{\alpha \in \Gamma} A_{\alpha}^{U}\right]$. 
Result 2.B[7, Theorem 1]. Let $A, B, C \in D(I)^{X}$ and let $\left\{A_{\alpha}\right\}_{\alpha \in \Gamma} \subset$ $D(I)^{X}$. Then:
(a) $\widetilde{0} \subset \mathrm{A} \subset \widetilde{1}$.
(b) $A \cup B=B \cup A, A \cap B=B \cap A$.
(c) $A \cup(B \cup C)=(A \cup B) \cup C, A \cap(B \cap C)=(A \cap B) \cap C$.
(d) $A, B \subset A \cup B, A \cap B \subset A, B$.
(e) $A \cap\left(\bigcup_{\alpha \in \Gamma} A_{\alpha}\right)=\bigcup_{\alpha \in \Gamma}\left(A \cap A_{\alpha}\right)$.
(f) $A \cup\left(\bigcap_{\alpha \in \Gamma} A_{\alpha}\right)=\bigcap_{\alpha \in \Gamma}\left(A \cup A_{\alpha}\right)$.
(g) $(\widetilde{0})^{c}=\widetilde{1},(\widetilde{1})^{c}=\widetilde{0}$.
(h) $\left(A^{c}\right)^{c}=A$.
(i) $\left(\bigcup_{\alpha \in \Gamma} A_{\alpha}\right)^{c}=\bigcap_{\alpha \in \Gamma} A_{\alpha}^{c},\left(\bigcap_{\alpha \in \Gamma} A_{\alpha}\right)^{c}=\bigcup_{\alpha \in \Gamma} A_{\alpha}^{c}$.

Definition 2.4[7]. Let $A \in D(I)^{X}$ and let $x_{M} \in \operatorname{IVF}_{\mathrm{P}}(\mathrm{X})$. Then:

(i) The set $\left\{x \in X: A^{U}(x)>0\right\}$ is called the support of $A$ and is denoted by $S(A)$.

(ii) $x_{M}$ said to belong to $A$, denoted by $x_{M} \in A$, if $M^{L} \leq A^{L}(x)$ and $M^{U} \leq A^{U}(x)$ for each $x \in X$.

It is obvious that $A=\bigcup_{x_{M} \in A} x_{M}$ and $x_{M} \in A$ if and only if $x_{M^{L}} \in A^{L}$ and $x_{M^{U}} \in A^{U}$.

Definition 2.5[7]. Let $f: X \rightarrow Y$ be a mapping, let $A \in D(I)^{X}$ and let $B \in D(I)^{Y}$. Then:

(i) the image of $A$ under $f$, denoted by $f(A)$, is an IVFS in $Y$ defined as follows: For each $y \in Y$,

$$
f(A)^{L}(y)=\left\{\begin{array}{lll}
\bigvee_{y=f(x)} A^{L}(x) & \text { if } & f^{-1}(y) \neq \varnothing \\
0 & \text { otherwise }
\end{array}\right.
$$

and

$$
f(A)^{U}(y)=\left\{\begin{array}{lll}
\bigvee_{y=f(x)} A^{U}(x) & \text { if } & f^{-1}(y) \neq \varnothing \\
0 & \text { otherwise. }
\end{array}\right.
$$


(ii) the preimage of $B$ under $f$, denoted by $f^{-1}(B)$, is an IVFS in $Y$ defined as follows: For each $y \in Y$,

$$
f^{-1}(B)^{L}(y)=\left(B^{L} \circ f\right)(x)=B^{L}(f(x))
$$

and

$$
f^{-1}(B)^{U}(y)=\left(B^{U} \circ f\right)(x)=B^{U}(f(x))
$$

It can be easily seen that $f(A)=\left[f\left(A^{L}\right), f\left(A^{U}\right)\right]$ and $f^{-1}(B)=$ $\left[f^{-1}\left(B^{L}\right), f^{-1}\left(B^{U}\right)\right]$.

Result 2.C[7, Theorem 2]. Let $f: X \rightarrow Y$ be a mapping and $g: Y \rightarrow Z$ be a mapping. Then:
(a) $f^{-1}\left(B^{c}\right)=\left[f^{-1}(B)\right]^{c}, \forall B \in D(I)^{Y}$.
(b) $[f(A)]^{c} \subset f\left(A^{c}\right), \forall A \in D(I)^{Y}$.
(c) $B_{1} \subset B_{2} \Rightarrow f^{-1}\left(B_{1}\right) \subset f^{-1}\left(B_{2}\right)$, where $B_{1}, B_{2} \in D(I)^{Y}$.
(d) $A_{1} \subset A_{2} \Rightarrow f\left(A_{1}\right) \subset f\left(A_{2}\right)$, where $A_{1}, A_{2} \in D(I)^{X}$.
(e) $f\left(f^{-1}(B)\right) \subset B, \forall B \in D(I)^{Y}$.
(f) $A \subset f\left(f^{-1}(A)\right), \forall A \in D(I)^{Y}$.
(g) $(g \circ f)^{-1}(C)=f^{-1}\left(g^{-1}(C)\right), \forall C \in D(I)^{Z}$.
(h) $f^{-1}\left(\bigcup_{\alpha \in \Gamma} B_{\alpha}\right)=\bigcup_{\alpha \in \Gamma} f^{-1} B_{\alpha}$, where $\left\{B_{\alpha}\right\}_{\alpha \in \Gamma} \in D(I)^{Y}$.
(h) $f^{-1}\left(\bigcap_{\alpha \in \Gamma} B_{\alpha}\right)=\bigcap_{\alpha \in \Gamma} f^{-1} B_{\alpha}$, where $\left\{B_{\alpha}\right\}_{\alpha \in \Gamma} \in D(I)^{Y}$.

\section{Interval-valued fuzzy subgroupoids}

Definition 3.1. Let $(X, \cdot)$ be a groupoid and let $A, B \in D(I)^{X}$. Then the interval-valued fuzzy product of $A$ and $B$, denoted by $A \circ B$, is an IVFS in $X$ defined as follows : For each $x \in X$,

$(A \circ B)(x)=\left\{\begin{array}{cl}{\left[\bigvee_{y z=x}\left[A^{L}(y) \wedge B^{L}(z)\right], \bigvee_{y z=x}\left[A^{U}(y) \wedge B^{U}(z)\right]\right]} & \text { if } y z=x, \\ 0 & \text { otherwise. }\end{array}\right.$

Definition 3.1'[8]. Let $X$, ० be geoupoid and let $A, B \in \operatorname{IFS}(X)$. Then the intuitionistic fuzzy product of $A$ and $B, A \circ B$, is defined as follow : For any $x \in X$, 
$\mu_{A \circ B}(x)=\left\{\begin{array}{llr}\left.\bigvee_{y z=x}\left[\mu_{A}(y) \wedge \mu_{B}(z)\right]\right] & \text { if } \quad \exists(y, z) \in X \times X \text { with } y z=x, \\ 0 & \text { otherwise. }\end{array}\right.$

and

$$
\nu_{A \circ B}(x)=\left\{\begin{array}{lr}
\bigwedge_{y z=x}\left[\nu_{A}(y) \vee \nu_{B}(z)\right] \quad \text { if } \quad \exists(y, z) \in X \times X \text { with } \quad y z=x \\
1 & \text { otherwise }
\end{array}\right.
$$

Remark 3.1. By Result 2.A, Definition 3.1 is reduced to Definition $3.1^{\prime}$ and the reverse holds.

Proposition 3.2. Let "o" be same as above, let $x_{M}, y_{N} \in \operatorname{IVFp}(X)$ and let $A, B \in D(I)^{X}$. Then:
(a) $x_{M} \circ y_{N}=(x y)_{M \cap N}$.
(a) $A \circ B=\bigcup_{x_{M} \in A, y_{N} \in B} x_{M} \circ y_{N}$.

Proof. (a) Let $z \in X$. Then

$$
\begin{gathered}
\left(x_{M} \circ y_{N}\right)(z)=\left\{\begin{array}{lr}
{\left[\bigvee_{z=x^{\prime} y^{\prime}}\left(x_{M}^{L}\left(x^{\prime}\right) \wedge y_{N}^{L}\left(y^{\prime}\right)\right), \bigvee_{z=x^{\prime} y^{\prime}}\left(x_{M}^{U}\left(x^{\prime}\right) \wedge y_{N}^{U}\left(y^{\prime}\right)\right)\right]} \\
0 & \text { if } x^{\prime} y^{\prime}=z, \\
\text { otherwise. }
\end{array}\right. \\
=\left\{\begin{array}{cc}
{\left[M^{L} \wedge N^{L}, M^{U} \wedge N^{U}\right]} & \text { if } z=x y, \\
0 & \text { otherwise. }
\end{array}\right. \\
=(x y)_{M \cap N}
\end{gathered}
$$

(b) Let $C=\bigcup_{x_{M} \in A, y_{N} \in B} x_{M} \circ y_{N}$, i.e.,

$$
C=\left[\bigwedge_{x_{M^{L}} \in A^{L}, y_{N} L \in B^{L}}\left(x_{M^{L}} \circ y_{N^{L}}\right), \bigwedge_{x_{M^{U}} \in A^{U}, y_{N^{U}} \in B^{U}}\left(x_{M^{U}} \circ y_{N^{U}}\right)\right] .
$$


For each $z \in X$, we may assume that $\exists u, v \in X$ such that $u v=z$, $x_{M}(u) \neq \mathbf{0}$ and $y_{N}(v) \neq \mathbf{0}$, without loss of generality. Then

$$
\begin{aligned}
& (A \circ B)^{L}(z)=\bigvee_{z=u v}\left[A^{L}(u) \wedge B^{L}(v)\right] \\
& \geq \bigvee_{z=u v}\left(\bigvee_{x_{M^{L}} \in A^{L}, y_{N} \in^{L}}\left[B_{M^{L}}(u) \wedge y_{N^{L}}(v)\right]\right) \\
& =\left(\bigcup_{x_{M^{L}} \in A^{L}, y_{N^{L}} \in B^{L}} x_{M^{L}} \circ y_{N^{L}}\right) \\
& =C^{L}(z) .
\end{aligned}
$$

Since $u_{A(u)} \in A$ and $v_{B(v)} \in B$,

$$
\begin{aligned}
& C^{L}(z)=\bigvee_{x_{M^{L}} \in A^{L}, y_{N} \in^{L}}\left(\bigvee_{z=u v}\left[x_{M^{L}}(u) \wedge y_{N^{L}}(v)\right]\right) \\
& =\bigvee_{z=u v}\left(\bigvee_{x_{M^{L}} \in A^{L}, y_{N} \in^{L}}\left[x_{M^{L}}(u) \wedge y_{N^{L}}(v)\right]\right) \\
& \geq \bigvee_{z=u v}\left[u_{A^{L}(u)}(u) \wedge v_{B^{L}(v)}(v)\right] \\
& =\bigvee_{z=u v}\left[A^{L}(u) \wedge B^{L}(v)\right] \\
& =(A \circ B)^{L}(z) .
\end{aligned}
$$

Thus $(A \circ B)^{L}=C^{L}$. By the similar arguments, we have $(A \circ B)^{U}=C^{U}$. Hence

$$
A \circ B=\bigcup_{x_{M^{L}} \in A^{L}, y_{N} L} x_{B^{L}} x_{M^{L}} \circ y_{N^{L}}
$$

The following is the immediate result of Definition 3.1.

Proposition 3.3. Let $(X, \circ)$ be a groupoid, and let "o" be same as above. $D(I)^{X}$

(a) if "०" is associative[resp. commutative] in $X$, the so is "o" in

(b) if "o" is has an identity $e \in X$, then $e_{\mathbf{1}} \in \operatorname{IVFp}(X)$ is an identity of "०" in $D(I)^{X}$, i.e., $A \circ e_{\mathbf{1}}=A=e_{\mathbf{1}} \circ A$ for each $A \in D(I)^{X}$.

Definition 3.4. Let $(G, \cdot)$ be a groupoid and let $\widetilde{0}=A \in D(I)^{X}$. Then $A$ is called an interval-valued fuzzy groupoid (in short, IVGP) in $G$ if 
$A \circ A \subset A$, i.e., $A^{L} \circ A^{L} \subset A^{L}$ and $A^{U} \circ A^{U} \subset A^{U}$.

We will denote the IVGPs in $G$ as $\operatorname{IVGP}(G)$.

Remark 3.4. (a) If $A$ is a fuzzy groupoid in a group $G$ in the sense of Liu[11], then $A=[A, A] \in \operatorname{IVGP}(G)$.

(b)If $A \in \operatorname{IVGP}(G)$, then $A^{L}, A^{U} \in \operatorname{FGP}(G)$ and $A_{*} \in \operatorname{IFGP}(G)$, where $\operatorname{FGP}(G)[\operatorname{resp}$. IFGP $(G)]$ denoted the set of all fuzzy groupoids in the sense of $\mathrm{Liu}[$ resp. the set of all intuitionistic fuzzy groupiods in the sense of Hur et al.].

The followings are the immediate results of Definitions 3.1 and 3.4.

Proposition 3.5. Let $(G, \cdot)$ be a groupoid and let $\widetilde{0} \neq A \in D(I)^{X}$. Then the followings are equivalent:

(a) $A \in \operatorname{IVGP}(G)$.

(b) For any $x_{M}, y_{N} \in A, x_{M} \circ y_{N} \in A$, i.e., $(A, \circ)$ is a groupoid.

(c) For any $x, y \in G, A^{L}(x y) \geq A^{L}(x) \wedge A^{L}(y)$ and $A^{U}(x y) \geq$ $A^{U}(x) \wedge A^{U}(y)$.

Proposition 3.6. Let $\widetilde{0} \neq A \in D(I)^{X}$. Then the followings are equivalent:

(a) If "०" is associative in $G$, then so is "०" in $A$, i.e., for any $x_{L}, y_{M}, z_{N} \in A$,

$x_{L} \circ\left(y_{M} \circ z_{N}\right)=\left(x_{L} \circ y_{M}\right) \circ z_{N}$.

(b) If "o" is commutative in $G$, then so is "o" in $A$, i.e., for any $x_{L}, y_{M} \in A$,

$x_{L} \circ y_{M}=y_{M} \circ x_{L}$.

(c) If "o" has an identity $e \in G$, then

$e_{\mathbf{1}} \circ x_{L}=x_{L}=x_{L} \circ e_{\mathbf{1}} \forall x_{L} \in A$.

From Proposition 3.5, we can define an IVGP in $G$ as follows.

Definition 3.4'. An interval-valued fuzzy set $A$ in $G$ is called an intervalvalued fuzzy subgroupoid(in short, IVGP) in $G$ if

$A^{L}(x y) \geq A^{L}(x) \wedge A^{L}(y)$ and $A^{U}(x y) \geq A^{U}(x) \wedge A^{U}(y), \forall x, y \in G$.

It is clear that $\widetilde{0}, \widetilde{1} \in \operatorname{IVGP}(G)$. 
The following is the immediate result of Definition $3.4^{\prime}$.

Proposition 3.7. Let $\mathrm{T} \in \mathrm{P}(G)$, where $\mathrm{P}(G)$ denoted the set of all subsets of $G$. Then $A=\left[\chi_{T}, \chi_{T}\right] \in \operatorname{IVGP}(G)$ if and only if T is a subgroupoid of $G$, where $\chi_{\mathrm{T}}$ is the charecteristic function of $\mathrm{T}$.

Proposition 3.8. If $\left\{A_{\alpha}\right\}_{\alpha \in \Gamma} \subset \operatorname{IVGP}(G)$, then $\bigcap_{\alpha \in \Gamma} A_{\alpha} \in \operatorname{IVGP}(G)$.

Proof. Let $A=\bigcap_{\alpha \in \Gamma} A_{\alpha}$ and let $x, y \in G$. Then

$$
\begin{aligned}
& A^{L}(x y)=\bigwedge_{\alpha \in \Gamma} A_{\alpha}^{L}(x y) \\
& \geq \bigwedge_{\alpha \in \Gamma}\left[A_{\alpha}^{L}(x) \wedge A_{\alpha}^{L}(y)\right]\left[\text { Since } A_{\alpha} \in \operatorname{IVGP}(G)\right] \\
& =\left(\bigwedge_{\alpha \in \Gamma} A_{\alpha}^{L}(x)\right) \wedge\left(\bigwedge_{\alpha \in \Gamma} A_{\alpha}^{L}(y)\right) \\
& =\left(\bigcap_{\alpha \in \Gamma} A_{\alpha}^{L}\right)(x) \wedge\left(\bigcap_{\alpha \in \Gamma} A_{\alpha}^{L}\right)(y) \\
& =A^{L}(x) \wedge A^{L}(y) .
\end{aligned}
$$

Similarly, we can see that $A^{U}(x y) \geq A^{U}(x) \wedge A^{U}(y)$. Hence $\bigcap_{\alpha \in \Gamma} A_{\alpha} \in$ $\operatorname{IVGP}(G)$.

Proposition 3.9. Let $f: G \rightarrow G^{\prime}$ be a groupoid homomorphism, let $A \in D(I)^{X}$ and let $B \in D(I)^{Y}$.
(a) $f\left(x_{M} \circ y_{N}\right)=f(x)_{M} \circ f(y)_{N}, \forall x_{M}, y_{N} \in \operatorname{IVFp}(G)$.
(b) If $f$ is surjective and $A \in \operatorname{IVGP}(G)$, then $f(A) \in \operatorname{IVGP}\left(G^{\prime}\right)$.
(c) If $B \in \operatorname{IVGP}\left(G^{\prime}\right)$, then $f^{-1}(B) \in \operatorname{IVGP}(G)$.

Proof. (a) Let $x_{M}, y_{N} \in \operatorname{IVP}(G)$ and let $z \in G^{\prime}$. Then

$$
\begin{aligned}
& f\left(x_{M} \circ y_{N}\right)^{L}(z)=f\left((x y)_{M^{L} \wedge N^{L}}\right)(z) \text { [By Proposition 3.2] } \\
& =\bigvee_{z^{\prime}=f(z)}(x y)_{M^{L} \wedge N^{L}}\left(z^{\prime}\right) \\
& =\left\{\begin{array}{crr}
M^{L} \wedge N^{L} & \text { if } & z^{\prime}=f(x y), \\
0 & & \text { otherwise. }
\end{array}\right.
\end{aligned}
$$

On the other hand,

$$
\left(f(x)_{M} \circ f(y)_{N}\right)^{L}(z)
$$




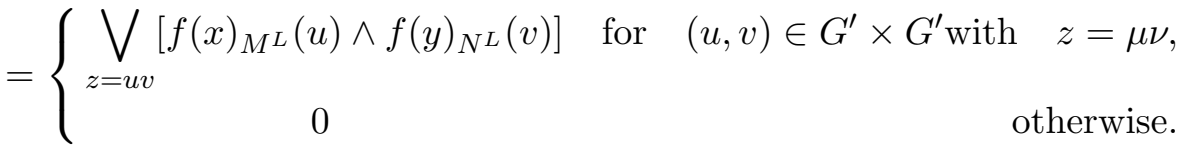

$$
\begin{aligned}
& =\left\{\begin{array}{crr}
M^{L} \wedge N^{L} & \text { if } & z=f(x) f(y), \\
0 & \text { otherwise. }
\end{array}\right.
\end{aligned}
$$

Thus $f\left(x_{M} \circ y_{N}\right)^{L}(z)=\left(f(x)_{M} \circ f(y)_{N}\right)^{L}(z)$. Similarly, we can see that $f\left(x_{M} \circ y_{N}\right)^{U}(z)=\left(f(x)_{M} \circ f(y)_{N}\right)^{U}(z), \forall z \in G^{\prime}$. So $f\left(x_{M} \circ y_{N}\right)=$ $f\left(x_{M}\right) \circ f\left(y_{N}\right)$.

(b) Assume that $f(A) \in \operatorname{IVGP}\left(G^{\prime}\right)$. Then $\exists y, y^{\prime} \in G^{\prime}$ such that

$$
f(A)^{L}\left(y y^{\prime}\right)<f(A)^{L}(y) \wedge f(A)^{L}\left(y^{\prime}\right)
$$

or

$$
f(A)^{U}\left(y y^{\prime}\right)<f(A)^{U}(y) \wedge f(A)^{U}\left(y^{\prime}\right) .
$$

Thus

$\mathrm{Or}$

$$
\bigvee_{f(z)=y y^{\prime}} A^{L}(z)<\left(\bigvee_{f(x)=y} A^{L}(x)\right) \wedge\left(\bigvee_{f\left(x^{\prime}\right)=y^{\prime}} A^{L}\left(x^{\prime}\right)\right)
$$

$$
\bigvee_{f(z)=y y^{\prime}} A^{U}(z)<\left(\bigvee_{f(x)=y} A^{U}(x)\right) \wedge\left(\bigvee_{f\left(x^{\prime}\right)=y^{\prime}} A^{U}\left(x^{\prime}\right)\right)
$$

Since $f$ is surjective, $\exists x, x^{\prime} \in G$ such that $f(x)=y, f\left(x^{\prime}\right)=y^{\prime}$, and

$$
\begin{aligned}
& \bigvee_{\text {or }} A^{L}(z)=y y^{\prime} \\
& \bigvee_{\text {So }} A^{U}(z)<A^{L}(z) \wedge A^{U}(x) \wedge A^{U}\left(x^{\prime}\right) . \\
& \\
& A^{L}\left(x x^{\prime}\right) \leq \bigvee_{f(z)=y y^{\prime}} A^{L}(z)<A^{L}(x) \wedge A^{L}\left(x^{\prime}\right) \\
& \text { or } \quad \\
& A^{U}\left(x x^{\prime}\right) \leq \bigvee_{f(z)=y y^{\prime}} A^{U}(z)<A^{U}(x) \wedge A^{U}\left(x^{\prime}\right) .
\end{aligned}
$$

This is a contradiction from the fact that $A \in \operatorname{IVGP}(G)$.

(c) It can be easily seen that $f^{-1}(B) \in \operatorname{IVGP}(G)$

Definition 3.10[2]. $A \in D(I)^{X}$ is said to have the sup-property if for each $T \in P(X), \exists t_{0} \in T$ such that $A\left(t_{0}\right)=\left[\bigvee_{t \in T} A^{L}(t), \bigwedge_{t \in T} A^{U}(t)\right]$. 
Definition 3.10' $[8] . \quad A \in \operatorname{IFS}(X)$ is said to have the sup-property if each $T \in P(X), \exists t_{0} \in T$ such that $A\left(t_{0}\right)=\left(\bigvee_{t \in T} \mu_{A}(t), \bigwedge_{t \in T} \nu_{A}(t)\right)$

Remark 3.10. (a) If $A \in I^{X}$ has the sup-property, $A=[A, A] \in$ $D(I)^{X}\left[\right.$ resp. $\left.A=\left(A, A^{c}\right) \in \operatorname{IFS}(X)\right]$ has the sup-property.

(b) If $A=\left[A^{L}, A^{U}\right] \in D(I)^{X}$ [resp. $\left.A=\left(\mu_{A}, \nu_{A}\right) \in \operatorname{IFS}(X)\right]$ has the sup-property, then $A^{L}$ and $A^{U} \in I^{X}\left[\right.$ resp. $\mu_{A}$ and $\left.\nu_{A}{ }^{c} \in I^{X}\right]$ have the sup-property.

Proposition 3.11. Let $f: G \rightarrow G^{\prime}$ be a groupoid homomorphism and let $A \in D(I)^{X}$ have the sup-property. If $A \in \operatorname{IVGP}(G)$, then $f(A) \in$ $\operatorname{IVGP}\left(G^{\prime}\right)$.

proof. Let $y, y^{\prime} \in G^{\prime}$. Then we can consider four cases:

(i) $f^{-1}(y) \neq \varnothing$ and $f^{-1}\left(y^{\prime}\right) \neq \varnothing$,

(ii) $f^{-1}(y) \neq \varnothing$ and $f^{-1}\left(y^{\prime}\right)=\varnothing$,

(iii) $f^{-1}(y)=\varnothing$ and $f^{-1}\left(y^{\prime}\right) \neq \varnothing$,

(iv) $f^{-1}(y)=\varnothing$ and $f^{-1}\left(y^{\prime}\right)=\varnothing$.

We prove only the case (i) and omit the remainders. Since $A$ has the sup-property, $\exists x_{0} \in f^{-1}(y)$ and $x_{0}^{\prime} \in f^{-1}\left(y^{\prime}\right)$ such that

and

$$
A\left(x_{0}\right)=\left[\bigvee_{t \in f^{-1}(y)} A^{L}(t), \bigvee_{t \in f^{-1}(y)} A^{U}(t)\right]
$$

$$
A\left(x_{0}^{\prime}\right)=\left[\bigvee_{t^{\prime} \in f^{-1}\left(y^{\prime}\right)} A^{L}\left(t^{\prime}\right), \bigvee_{t^{\prime} \in f^{-1}\left(y^{\prime}\right)} A^{U}\left(t^{\prime}\right)\right] .
$$

Then

$$
\begin{aligned}
& f(A)^{L}\left(y y^{\prime}\right)=\bigvee_{z \in f^{-1}\left(y y^{\prime}\right)} A^{L}(z) \geq A^{L}\left(x_{0} x_{0}^{\prime}\right)\left[\text { Since } f\left(x_{0} x_{0}^{\prime}\right)=f\left(x_{0}\right) f\left(x_{0}^{\prime}\right)\right. \\
& \left.=y y^{\prime}\right] \\
& \geq A^{L}\left(x_{0}\right) \wedge A^{L}\left(x_{0}^{\prime}\right)[\text { Since } A \in \operatorname{IVGP}(G) .] \\
& =\left(\bigvee_{t \in f^{-1}(y)} A^{L}(t)\right) \wedge\left(\bigvee_{t^{\prime} \in f^{-1}\left(y^{\prime}\right)} A^{L}\left(t^{\prime}\right)\right) \\
& =f(A)^{L}(y) \wedge f(A)^{L}\left(y^{\prime}\right) .
\end{aligned}
$$

Similarly, we have $f(A)^{U}\left(y y^{\prime}\right) \geq f(A)^{U}(y) \wedge f(A)^{U}\left(y^{\prime}\right)$. So $f(A) \in$ $\operatorname{IVGP}\left(G^{\prime}\right)$.

Definition 3.12. Let $f: X \rightarrow Y$ be a mapping and let $A \in D(I)^{X}$. Then $A$ is said to be interval-valued fuzzy invariant(in short, IVFinvariant) if $f(x)=f(y)$ implies $A(x)=A(y)$, i.e., $A^{L}(x)=A^{L}(y)$ 
and $A^{U}(x)=A^{U}(y)$.

It is clear that if $A$ is IVF-invariant, i.e., $f^{-1}(f(A))=A$.

The following is the immediate result of Definition 3.12.

Proposition 3.13. Let $f: X \rightarrow Y$ be a mapping and let $\mathcal{A}=\{A \in$ $D(I)^{X}: A$ is IVF-invariant and has the sup-property $\}$. Then there is a one-to-one correspondence between $\mathcal{A}$ and $D(I)^{\operatorname{Im} f}$, where $\operatorname{Im} f$ denotes the image of $f$

The following is the immediatd result of Propositions 3.11 and 3.13.

Corollary 3.13. Let $f: G \rightarrow G^{\prime}$ be a groupoid homomorphism and let $\mathcal{A}=\{A \in \operatorname{IVGP}(G): A$ is IVF-invariant and has the sup-property $\}$. Then there is a one-to-one correspondence between $\mathcal{A}$ and IVGP $(\operatorname{Im} f)$.

\section{Interval-value fuzzy subgroups}

Definition 4.1[4]. Let $A$ be an $I V F s$ in a group $G$. Then $A$ is called an interval-valued fuzzy subgroup (in short, $I V G$ ) in $G$ if it satisfies the conditions : For any $x, y \in G$,

(i) $A^{L}(x y) \geq A^{L}(x) \wedge A^{L}(y)$ and $A^{U}(x y) \geq A^{U}(x) \wedge A^{U}(y)$

(ii) $A^{L}\left(x^{-1}\right) \geq A^{L}(x)$ and $A^{U}\left(x^{-1}\right) \geq A^{U}(x)$

We will denote the set of all IVGS of G as $\operatorname{IVG}(G)$.

Example 4.1. Consider the additive group $(\mathbb{Z},+)$. We define a mapping $A=\left[A^{L}, A^{U}\right]: \mathbb{Z} \rightarrow D(I)$ as follows : For each $n \in \mathbb{Z}$.

$A(0)=\left[A^{L}(0), A^{U}(0)\right]=[1,1]$,

and

$$
A(n)=\left[A^{L}(n), A^{U}(n)\right]= \begin{cases}{\left[\frac{1}{2}, \frac{2}{3}\right],} & \text { if } \mathrm{n} \text { is odd }, \\ {\left[\frac{1}{3}, \frac{4}{5}\right],} & \text { if } \mathrm{n} \text { is even. }\end{cases}
$$

Then clearly $A \in D(I)^{\mathbb{Z}}$. Moreover, $A$ satisfies all the conditions of Definition 4.1. So $A \in \operatorname{IVG}(\mathbb{Z})$. 
Remark 4.1. (a) If $A \in \mathrm{FG}(\mathrm{G})$, then $A=[A, A] \in \operatorname{IVG}(\mathrm{G})$, where $\mathrm{FG}(G)$ denotes the set of all fuzzy groups in $G$.

(b) If $A \in \operatorname{IVG}(\mathrm{G})$, then $A^{L}, A^{U} \in \mathrm{FG}(\mathrm{G})$ and $\left(A^{L}, A^{U^{C}}\right) \in \operatorname{IFG}(\mathrm{G})$.

(c) If $A \in \operatorname{IFG}(\mathrm{G})$, then $\left[\mu_{A}, \nu_{A}^{c}\right] \in \operatorname{IVG}(\mathrm{G})$.

The following two results can be easily proved from definition 4.1, Propositions 3.7 and 3.8 .

Proposition 4.2. Let $\mathrm{G}$ be a group and let $H \subset G$. Then $H$ is a subgroup of $\mathrm{G}$ if and only if $\left[\chi_{H}, \chi_{H}\right] \in \operatorname{IVG}(\mathrm{G})$.

Proposition 4.3. Let $\left\{A_{\alpha}\right\}_{\alpha \in \Gamma} \subset \operatorname{IVG}(\mathrm{G})$. Then $\bigcap_{\alpha \in \Gamma} A_{\alpha} \in \operatorname{IVG}(\mathrm{G})$.

The followings can be easily seen from Definitions 3.1 and 4.1.

Proposition 4.4. Let $G$ be group and let $A \in D(I)^{G}$. If $A \in \operatorname{IVG}(\mathrm{G})$, then $A \circ A=A$.

Proposition 4.5. Let $A, B \in \operatorname{IVG}(\mathrm{G})$. Then $A \circ B \in \operatorname{IVG}(\mathrm{G})$ if and only if $A \circ B=B \circ A$.

Result 4.A [4, Proposition 3.1]. Let $A$ be an IVG in a group $G$.

(a) $A\left(x^{-1}\right)=A(x), \forall x \in G$.

(b) $A^{L}(e) \geq A^{L}(x)$ and $A^{U}(e) \geq A^{U}(x), \forall x \in G$, where $e$ is the identity of $G$.

Result 4.B [4, Proposition 3.2]. Let $A$ be an IVFS in a group $G$. Then $A$ is an IVG in $G$ if and only if $A^{L}\left(x y^{-1}\right) \geq A^{L}(x) \wedge A^{L}(y)$ and $A^{U}\left(x y^{-1}\right) \geq A^{U}(x) \wedge A^{U}(y), \forall x, y \in G$.

Proposition 4.6. If $A \in \mathrm{IVG}(\mathrm{G})$, then $G_{A}=\{x \in G: \mathrm{A}(x)=\mathrm{A}(\mathrm{e})\}$ is a subgroup of $G$.

Proof. let $x, y \in G_{A}$. Then

$$
\begin{aligned}
& A^{L}\left(x y^{-1}\right) \geq A^{L}(x) \wedge A^{L}\left(y^{-1}\right) \\
& =A^{L}(x) \wedge A^{L}(y)[\text { By Result } 4 . \mathrm{A}] \\
& =A^{L}(e) \wedge A^{L}(e)\left[\text { Since } x, y \in G_{A}\right] \\
& =A^{L}(e) .
\end{aligned}
$$

Similarly, we have $A^{U}\left(x y^{-1}\right) \geq A^{U}(e)$, On the other hand, by Result 4.A, it is clear that $A^{L}\left(x y^{-1}\right) \leq A^{L}(e)$ and $A^{U}\left(x y^{-1}\right) \leq A^{U}(e)$, thus 
$A\left(x y^{-1}\right)=A(e)$. So $x y^{-1} \in G_{A}$. Hence $G_{A}$ is a subgroup of $G$.

Proposition 4.7. let $A \in \operatorname{IVG}(\mathrm{G})$. If $A\left(x y^{-1}\right)=A(e)$ for any $x, y \in G$, then $A(x)=A(y)$.

Proof. Let $x, y \in G$. Then

$A^{L}(x)=A^{L}\left(\left(x y^{-1}\right) y\right)$

$\geq A^{L}\left(x y^{-1}\right) \wedge A^{L}(y)[$ Since $A \in \operatorname{IVG}(\mathrm{G})]$

$=A^{L}(e) \wedge A^{L}(y)[$ By the hypothesis ]

$=A^{L}(y)$. [ By Result 4.A. ]

On the other hard, by Result 4.A, $A^{L}\left(x^{-1}\right)=A^{L}(x)$. Then

$A^{L}(y)=A^{L}\left(\left(y x^{-1}\right) x\right)$

$\geq A^{L}\left(y x^{-1}\right) \wedge A^{L}(x)$

$=A^{L}\left(\left(y x^{-1}\right)^{-1}\right) \wedge A^{L}(x)$ [ By Result 4.A. ]

$=A^{L}\left(x y^{-1}\right) \wedge A^{L}(x)$

$=A^{L}(e) \wedge A^{L}(x)$

$=A^{L}(x)$.

Similarly, we have $A^{U}(x)=A^{U}(y)$. Hence $A(x)=A(y)$.

Corollary 4.7-1. Let $A \in \operatorname{IVG}(\mathrm{G})$. If $G_{A}$ is a normal subgroup of $G$, then $A$ is constant on each coset of $G_{A}$.

Proof. Let $a \in G$ and let $x \in a G_{A}$. Then $\exists y \in G_{A}$ such that $x=a y$. Since $G_{A}$ is normal, $x a^{-1} \in G_{A}$. Thus, by the definition of $G_{A}, A\left(x a^{-1}\right)=A(e)$. By proposition $4.7, A(x)=A(a)$. So $A$ is constant on $a G_{A} \forall a \in G$. Similarly, we can see that $A$ is constant on $G_{A} a \forall a \in G$. This completes the proof.

Let $H$ be a subgroup of $G$. Then the number of right [resp. left] cosets of $H$ in $G$ is called the index of $H$ in $G$ and denoted by $[G: H]$. If $G$ is a finite group, then there can be only a finite number of distinct right [resp. left] cosets of $H$; hence the index $[G: H]$ is finite. If $G$ is an infinite group, then $[G: H]$ may be either finite or infinite.

Corollary 4.7-2. Let $A \in \operatorname{IVG}(\mathrm{G})$ and let $G_{A}$ be normal. If $G_{A}$ has a finite index, then $A$ has the sup property.

Proof. Let $T \subset G$. Since $G_{A}$ has finite index, let the index $[G$ : $\left.G_{A}\right]=n$, say $\mathcal{A}=\left\{a_{1} G_{A}, \cdots, a_{n} G_{A}\right\}$, where $a_{i} \in G(i=1, \cdots, n)$ and $a_{i} G_{A} \cap a_{j} G_{A}=\tilde{0}$ for any $i \neq j$. Let $t \in T$. Since $G=\bigcup \mathcal{A}=\bigcup_{i=1}^{n} a_{i} G_{i}$, 
there exists an $i \in\{1, \cdots, n\}$ such that $t \in a_{i} G_{A}$. Since $G_{A}$ is normal, by Corollary 4.7-1, $A(t)=A\left(a_{i}\right)$ on $a_{i} G_{A}$, say $A^{L}(t)=\alpha_{i}$ and $A^{U}(t)=\beta_{i}$, where $\alpha_{i}, \beta_{i} \in I$ and $\alpha_{i} \leq \beta_{i}$. Thus there exists a $t_{0} \in T$ such that $A^{L}\left(t_{0}\right)=\bigvee_{t \in T} A^{L}(t)=\bigvee_{i=1}^{n} \alpha_{i}$ and $A^{U}\left(t_{0}\right)=\bigvee_{t \in T} A^{U}(t)=\bigvee_{i=1}^{n} \beta_{i}$. Hence $A$ has the sup property.

Proposition 4.8. A group $G$ cannot be the union of two proper IVGs.

Proof. Let $A$ and $B$ be proper IVGs of a group $G$ such that $A \cup B=\tilde{1}$, $A \neq \tilde{1}$ and $B \neq \tilde{1}$. Since $A \cup B=\left(A^{L} \vee B^{L}, A^{U} \vee B^{U}\right), A^{L}(x) \vee B^{L}(x)$ $=1$ and $A^{U}(x) \vee B^{U}(x)=1, \forall x \in X$. Then $A^{L}(x)=1$ or $B^{L}(x)=1$ and $A^{U}(x)=1$ or $B^{U}(x)=1$. Since $A \neq \tilde{1}$ and $B \neq \tilde{1}, A^{L}(x) \neq 1$ or $A^{U}(x) \neq 1$ and $B^{L}(x) \neq 1$ or $B^{U}(x) \neq 1$. In either cases, this is a contradiction. This completes the proof.

Proposition 4.9. If $A$ is an IVGP of a finite group $G$, then $A \in \operatorname{IVG}(\mathrm{G})$.

Proof. Let $x \in G$. Since $G$ is finite, $x$ has the finite order, say $n$, Then $x^{n}=e$, where $e$ is the identity of $G$. Thus $x^{-1}=x^{n-1}$. Since $A$ is an IVGP of $G$,

and

$$
A^{L}\left(x^{-1}\right)=A^{L}\left(x^{n-1}\right)=A^{L}\left(x^{n-2} x\right) \geq A^{L}(x)
$$

$$
A^{U}\left(x^{-1}\right)=A^{U}\left(x^{n-1}\right)=A^{U}\left(x^{n-2} x\right) \geq A^{U}(x) .
$$

Hence $A \in \operatorname{IVG}(\mathrm{G})$.

Proposition 4.10. Let $A$ be an IVG of a group $G$ and let $x \in G$. Then $A(x y)=A(y)$, for each $y \in G$ if and only if $A(x)=A(e)$.

Proof. $(\Rightarrow)$ :Suppose $A(x y)=A(y)$ for each $y \in G$. Then clearly $A(x)=A(e)$.

$(\Leftarrow)$ :Suppose $A(x)=A(e)$. Then, by Result 4 .A, $A^{L}(y) \leq A^{L}(x)$ and $A^{U}(y) \leq A^{U}(x)$ for each $y \in G$. Since $A$ is an IVG of $G$, Then $A^{L}(x y) \geq$ $A^{L}(x) \wedge A^{L}(y)$ and $A^{U}(x y) \geq A^{U}(x) \vee A^{U}(y)$. Thus $A^{L}(x y) \geq A^{L}(y)$ and $A^{U}(x y) \geq A^{U}(y)$ for each $y \in G$.

On the other hand, by Result 4.A, and

$$
A^{L}(y)=A^{L}\left(x^{-1} x y\right) \geq A^{L}(x) \wedge A^{L}(x y)
$$

$$
A^{U}(y)=A^{U}\left(x^{-1} x y\right) \geq A^{U}(x) \wedge A^{U}(x y) .
$$

Since $A^{L}(x) \geq A^{L}(y)$ for each $y \in G, A^{L}(x) \wedge A^{L}(x y)=A^{L}(x y)$ and $A^{U}(x) \wedge A^{L}(x y)=A^{U}(x y)$. So $A^{L}(y) \geq A^{L}(x y)$ and $A^{U}(y) \geq A^{U}(x y)$ 
for each $y \in G$. Hence $A(x y)=A(y)$ for each $y \in G$.

Proposition 4.11. Let $f: G \rightarrow G^{\prime}$ be a group homomorphism, let $A \in$ $\operatorname{IVG}(G)$ and let $B \in \operatorname{IVG}\left(G^{\prime}\right)$. Then the following hold:

(a) If $A$ has the sup property, then $f(A) \in \operatorname{IVG}\left(G^{\prime}\right)$.

(b) $f^{-1}(B) \in \operatorname{IVG}(G)$.

Proof. (a) By Proposition 3.11, since $f(A) \in \operatorname{IVGP}(G)$, it is enough to show that $f(A)^{L}\left(y^{-1}\right) \geq f(A)^{L}(y)$ and $f(A)^{U}\left(y^{-1}\right) \geq f(A)^{U}(y)$ for each $y \in f(G)$.

Let $y \in f(G)$. Then $\phi \neq f^{-1}(y) \subset G$. Since $A$ has the sup property, there exists an $x_{0} \in f^{-1}(y)$ such that $A^{L}\left(x_{0}\right)=\bigvee_{t \in f^{-1}(y)} A^{L}(t)$ and $A^{U}\left(x_{0}\right)=\bigvee_{t \in f^{-1}(y)} A^{U}(t)$.

Thus

$$
f(A)^{L}\left(y^{-1}\right)=\bigvee_{t \in f^{-1}\left(y^{-1}\right)} A^{L}(t) \geq A^{L}\left(x_{0}^{-1}\right) \geq A^{L}\left(x_{0}\right)=f(A)^{L}(y)
$$

and

$$
f(A)^{U}\left(y^{-1}\right)=\bigvee_{t \in f^{-1}\left(y^{-1}\right)} A^{U}(t) \geq A^{U}\left(x_{0}^{-1}\right) \geq A^{U}\left(x_{0}\right)=f(A)^{U}(y) .
$$

Hence $f(A) \in \operatorname{IVG}(G)$.

(b) By proposition 3.9, since $f^{-1}(B) \in \operatorname{IVGP}(G)$, it is enough to show that $f^{-1}(B)^{L}\left(x^{-1}\right) \geq f^{-1}(B)^{L}(x)$ and $f^{-1}(B)^{U}\left(x^{-1}\right) \geq f^{-1}(B)^{U}(x)$ for each $x \in G$.

Let $x \in G$. Then

$$
\begin{aligned}
& f^{-1}(B)^{L}\left(x^{-1}\right)=B^{L}\left(f\left(x^{-1}\right)\right)=B^{L}\left(f(x)^{-1}\right) \\
& \geq B^{L}(f(x))=f^{-1}(B)^{L}(x)
\end{aligned}
$$

and

$$
\begin{aligned}
& f^{-1}(B)^{U}\left(x^{-1}\right)=B^{U}\left(f\left(x^{-1}\right)\right)=B^{U}\left(f(x)^{-1}\right) \\
& \geq B^{U}(f(x))=f^{-1}(B)^{U}(x) .
\end{aligned}
$$

Thus $f^{-1}(B) \in \operatorname{IVG}(G)$. This completes the proof.

Proposition 4.12. Let $G_{p}$ be the cyclic group of prime order $p$. Then $A \in \operatorname{IVG}\left(G_{p}\right)$ if and only if $A^{L}(x)=A^{L}(1) \leq A^{L}(0)$ and $A^{U}(x)=$ $A^{U}(1) \leq A^{U}(0)$ for each $0 \neq x \in G_{p}$. 
Proof. $(\Rightarrow)$ : Suppose $A \in \operatorname{IVG}\left(G_{p}\right)$ and let $0 \neq x \in G_{p}$. Then $A^{L}(x y) \geq A^{L}(x) \wedge A^{L}(y)$ and $A^{U}(x y) \geq A^{U}(x) \wedge A^{U}(y)$ for any $x, y \in G_{p}$. Since $G_{p}$ is the cyclic group of prime order $p, G_{p}=\{0,1,2, \cdots, p-1\}$. Since $x$ is the sum of 1 's and 1 is the sum of $x^{\prime} s, A^{L}(x) \geq A^{L}(1) \geq A^{L}(x)$ and $A^{U}(x) \geq A^{U}(1) \geq A^{U}(x)$. Thus $A^{L}(x)=A^{L}(1)$ and $A^{U}(x)=$ $A^{U}(1)$. Since 0 is the identity element of $G_{p}, A^{L}(x) \leq A^{L}(0)$ and $A^{U}(x) \leq$ $A^{U}(0)$. Hence the necessary conditions hold.

$(\Leftarrow)$ : Suppose the necessary conditions hold and let $x, y \in G_{p}$. Then we have four cases: (i) $x \neq 0, y \neq 0$ and $x=y$, (ii) $x \neq 0, y=0$, (iii) $x=0, y \neq 0$, (iv) $x \neq 0, y \neq 0$ and $x \neq y$.

Case(i) Suppose $x \neq 0, y \neq 0$ and $x=y$. Then, by the hypothesis, $A^{L}(x)=A^{L}(y)=A^{L}(1) \leq A^{L}(0)$ and $A^{U}(x)=A^{U}(y)=A^{U}(1) \leq$ $A^{U}(0)$. So $A^{L}(x-y)=A^{L}(0) \geq A^{L}(x) \wedge A^{L}(y)$ and $A^{L}(x-y) \geq$ $A^{U}(x) \wedge A^{U}(y)$.

Case(ii) Suppose $x \neq 0$ and $y=0$. Since $x-y \neq 0$, by the hypothesis, $A^{L}(x-y)=A^{L}(x)=A^{L}(1) \leq A^{L}(0)=A^{L}(y)$ and $A^{U}(x-y)=$ $A^{U}(x)=A^{U}(1) \leq A^{U}(0)=A^{U}(y)$. So $A^{L}(x-y) \geq A^{L}(x) \wedge A^{L}(y)$ and $A^{U}(x-y) \geq A^{U}(x) \wedge A^{U}(y)$.

Case(iii) is the same as Case(ii).

Case(iv) Suppose $x \neq 0, y \neq 0$ and $x \neq y$. Since $x-y \neq 0$, by the hypothesis, $A^{L}(x-y)=A^{L}(x)=A^{L}(y)=A^{L}(1) \leq A^{L}(0)$ and $A^{U}(x-y)=A^{U}(x)=A^{U}(y) \leq A^{U}(0)$. So $A^{L}(x-y) \geq A^{L}(x) \wedge A^{L}(y)$ and $A^{U}(x-y) \geq A^{U}(x) \wedge A^{U}(y)$. In all, $A^{L}(x-y) \geq A^{L}(x) \wedge A^{L}(y)$ and $A^{U}(x-y) \geq A^{U}(x) \wedge A^{U}(y)$. Hence, by Result 4.B, $A \in \operatorname{IFG}\left(G_{p}\right)$.

Definition 4.13. Let $G$ be a groupoid and let $A \in \operatorname{IVS}(G)$. Then $A$ is called an:

(1) interval-valued fuzzy left ideal (in short, $I V L I$ ) of $G$ if for any $x, y \in G, A^{L}(x y) \geq A^{L}(y)$ and $A^{U}(x y) \geq A^{U}(y)$.

(2) interval-valued fuzzy right ideal (in short, IVRI) of $G$ if for any $x, y \in G, A^{L}(x y) \geq A^{L}(x)$ and $A^{U}(x y) \geq A^{U}(x)$.

(3) interval-valued fuzzy ideal (in short, $I V I$ ) of $G$ if it is both an IFLI and an IFRI.

We will denote the set of all IVLIs[resp. IVRIs and IVIs] of a groupiod $G$ as $\operatorname{IVLI}(\mathrm{G})[\operatorname{resp} . \operatorname{IVRI}(\mathrm{G})$ and $\operatorname{IVI}(\mathrm{G})]$.

It is clear that $A \in \operatorname{IVI}(\mathrm{G})$ if and only if and only if for any $x, y \in$ $G, A^{L}(x y) \geq A^{L}(x) \vee A^{L}(y)$ and $A^{U}(x y) \geq A^{U}(x) \vee A^{U}(y)$. Moreover, an IFI(resp. IFLI, IFRI) is an IVGP of $G$. Note that for any $A \in \operatorname{IVGP}(\mathrm{G})$, 
we have $A^{L}\left(x^{n}\right) \geq A^{L}(x)$ and $A^{U}\left(x^{n}\right) \geq A^{U}(x)$ for each $x \in G$, where $x^{n}$ is any composite of $x^{\prime} s$.

Proposition 4.14. The IVLIs (resp. IVLIs, IVRIs) in a group $G$ are just the constant mappings.

Proof. Suppose $A$ is an constant mapping and let $x, y \in G$. Then $A(x y)=A(x)=A(y)$. Thus $A \in \mathrm{IVI}(\mathrm{G})$.

Now suppose $A \in \operatorname{IVLI}(\mathrm{G})$. Then $A^{L}(x y) \geq A^{L}(y)$ and $A^{U}(x y) \geq$ $A^{U}(y)$ for any $x, y \in G$. In particular, $A^{L}(x) \geq A^{L}(e)$ and $A^{U}(x) \geq$ $A^{U}(e)$ for each $x \in G$. Moreover, $A^{L}(e)=A^{L}\left(x^{-1} x\right) \geq A^{L}(x)$ and $A^{U}(e)=A^{U}\left(x^{-1} x\right) \geq A^{U}(x)$ for each $x \in G$. So $A(x)=A(e)$ for each $x \in G$. Hence $A$ is a constant mapping.

Definition 4.15. Let $A$ be an IVFS in a set $X$ and let $\lambda, \mu \in I$ with $\lambda \leq \mu$. Then the set $A^{[\lambda, \mu]}=\left\{x \in X: A^{L}(x) \geq \lambda\right.$ and $\left.A^{U}(x) \geq \mu\right\}$ is called a $[\lambda, \mu]$-level subset of $A$.

Proposition 4.16. Let $A$ be an IVG of a group $G$. Then, for each $(\lambda, \mu) \in I \times I$ such that $\lambda \leq \mu_{A}(e), \mu \leq \nu_{A}(e)$ and $\lambda \leq \mu, A^{[\lambda, \mu]}$ is a subgroup of $G$.

Proof. Clearly, $A^{[\lambda, \mu]} \neq \emptyset$. Let $x, y \in A^{[\lambda, \mu]}$. Then $A^{L}(x) \geq \lambda, A^{U}(y) \geq$ $\mu$ and $A^{L}(y) \geq \lambda, A^{U}(y) \geq \mu$. Since $A \in \operatorname{IVG}(G), A^{L}(x y) \geq A^{L}(x) \wedge$ $A^{L}(y) \geq \lambda$ and $A^{U}(x y) \geq A^{U}(x) \wedge A^{U}(y) \geq \mu$. Thus $A^{L}(x y) \geq \lambda$ and $A^{U}(x y) \geq \mu$. So $x y \in A^{[\bar{\lambda}, \mu]}$. On the other hand, $A^{L}\left(x^{-1}\right) \geq A^{L}(x) \geq \lambda$ and $A^{U}\left(x^{-1}\right) \geq A^{U}(x) \geq \mu$. Thus $A^{L}\left(x^{-1}\right) \lambda$ and $A^{U}\left(x^{-1}\right) \geq \mu$. So $x^{-1} \in A^{[\lambda, \mu]}$. Hence $A^{[\lambda, \mu]}$ is a subgroup of $G$.

Proposition 4.16. Let $A$ be an IVS in a group $G$ such that $A^{[\lambda, \mu]}$ is a subgroup of $G$ for each $(\lambda, \mu) \in I \times I$ such that $\lambda \leq A^{L}(e), \mu \leq A^{U}(e)$ and $\lambda \leq \mu$. Then $A$ is an IVG of $G$.

Proof. For any $x, y \in G$, let $A(x)=\left[t_{1}, s_{1}\right]$ and let $A(y)=\left[t_{2}, s_{2}\right]$. Then clearly, $x \in A^{\left[t_{1}, s_{1}\right]}$ and $y \in A^{\left[t_{2}, s_{2}\right]}$. Suppose $t_{1}<t_{2}$ and $s_{1}<s_{2}$. Then $A^{\left[t_{2}, s_{2}\right]} \subset A^{\left[t_{1}, s_{1}\right]}$. Thus $y \in A^{\left[t_{1}, s_{1}\right]}$. Since $A^{\left[t_{1}, s_{1}\right]}$ is a subgroup of $G, x y \in A^{\left[t_{1}, s_{1}\right]}$. Then $A^{L}(x y) \geq t_{1}$ and $A^{U}(x y) \geq s_{1}$. So $A^{L}(x y) \geq A^{L}(x) \wedge A^{L}(y)$ and $A^{U}(x y) \geq A^{U}(x) \wedge A^{U}(y)$. For each $x \in G$, let $A(x y)=[\lambda, \mu]$. Then $x \in A^{[\lambda, \mu]}$. Since $A^{[\lambda, \mu]}$ is a subgroup of $G, x^{-1} \in A^{[\lambda, \mu]}$. So $A^{L}\left(x^{-1}\right) \geq A^{L}(x)$ and $A^{U}\left(x^{-1}\right) \geq A^{U}$. Hence $A \in$ 
$\operatorname{IVG}(G)$.

\section{Interval-value fuzzy normal subgroups}

Definition 5.1. Let $A \in \operatorname{IVG}(G)$. Then $A$ is called an intervalvalued fuzzy

normal subgroup (in short, $I V N G$ ) of $G$ if $A(x y)=A(y x)$, for any $x, y \in G$.

We will denote the set of all IVNGs of a group $G$ as $\operatorname{IVNG}(G)$. It is clear that if $G$ is abelian, then $A \in \operatorname{IVNG}(G), \forall A \in \operatorname{IVG}(G)$.

Example 5.1. Consider the general linear group of degree $n, G L(n, R)$. Then clearly, $G L(n, R)$ is a non abelian group. Let us define a mapping $A: G L(n, R) \rightarrow D(I)$ as follows: for any $I_{n} \neq M \in G L(n, R)$, where $I_{n}$ is the unit matrix,

$$
A\left(I_{n}\right)=\tilde{1}
$$

$$
A^{L}(M)= \begin{cases}\frac{1}{5} & \text { if } \mathrm{M} \text { is not a triangular matrix } \\ \frac{1}{3} & \text { if } \mathrm{M} \text { is a triangular matrix }\end{cases}
$$

and

$$
A^{U}(M)= \begin{cases}\frac{2}{3} & \text { if } \mathrm{M} \text { is not a triangular matrix, } \\ \frac{1}{2} & \text { if } \mathrm{M} \text { is a triangular matrix }\end{cases}
$$

Then we can easily see that $A$ is an IVNG of $G L(n, R)$.

The following is the immediate result of Definitions 3.1 and 5.1.

Proposition 5.2. Let $A \in D(I)^{G}$ and let $B \in \operatorname{IVNG}(G)$. Then $A \circ B=B \circ A$.

Proposition 5.3. Let $A \in \operatorname{IVNG}(G)$. If $B \in \operatorname{IVG}(G)$, then so is $B \circ A$.

Proof. By Definitions 3.1 and 3.4, it can be easily seen that $B \circ A \in$ $\operatorname{IVGP}(G)$. Thus it is sufficient to show that $(B \circ A)^{L}\left(x^{-1}\right) \geq(B \circ A)^{L}(x)$ and $(B \circ A)^{U}\left(x^{-1}\right) \geq(B \circ A)^{U}(x)$ for each $x \in G$. 
Let $x \in G$. Then

$$
\begin{aligned}
& (B \circ A)^{L}\left(x^{-1}\right)=\bigvee_{y z=x^{-1}}\left[B^{L}(y) \wedge A^{L}(z)\right] \\
& =\bigvee_{z^{-1} y^{-1}=x}\left[B^{L}\left(\left(y^{-1}\right)^{-1}\right) \wedge A^{L}\left(\left(z^{-1}\right)^{-1}\right)\right] \\
& \geq \bigvee_{z^{-1} y^{-1}=x}\left[B^{L}\left(y^{-1}\right) \wedge A^{L}\left(z^{-1}\right)\right] \\
& =(A \circ B)^{L}(x)=(B \circ A)^{L}(x) .
\end{aligned}
$$

Similarly, we have $(B \circ A)^{U}\left(x^{-1}\right) \geq(B \circ A)^{U}(x)$ for each $x \in G$. Hence $B \circ A \in \operatorname{IVG}(G)$.

Corollary 5.3. Let $A, B \in \operatorname{IVNG}(G)$. Then $A \circ B \in \operatorname{IVNG}(G)$.

Proof. By Proposition 4.5, $A \circ B \in \operatorname{IVG}(G)$. Let $a, b \in G$. Then there exists $x, y \in G$ such that $a b=x y$. Since $b=a^{-1} x y, b a=$ $\left(a^{-1} x a\right)\left(a^{-1} y a\right)$. Since $A, B \in \operatorname{IVNG}(G)$,

$$
\begin{aligned}
& (A \circ B)(a b)=\left[(A \circ B)^{L}(a b),(A \circ B)^{U}(a b)\right] \\
& =\left[\bigvee_{a b=x y}\left(A^{L}(x) \wedge B^{L}(y)\right), \bigvee_{a b=x y}\left(A^{U}(x) \wedge B^{U}(y)\right)\right] \\
& =\left[\bigvee_{b a=\left(a^{-1} x a\right)\left(a^{-1} y a\right)}\left(A^{L}\left(a^{-1} x a\right) \wedge B^{L}\left(a^{-1} y a\right)\right),\right. \\
& \\
& \bigvee_{b a=\left(a^{-1} x a\right)\left(a^{-1} y a\right)}\left(A^{U}\left(a^{-1} x a\right) \wedge B^{U}\left(a^{-1} y a\right)\right] \\
& =\left[(A \circ B)^{L}(b a),(A \circ B)^{U}(b a)\right] \\
& =(A \circ B)(b a) .
\end{aligned}
$$

Hence $A \circ B \in \operatorname{IFNG}(G)$.

Proposition 5.4. If $A \in \operatorname{IVNG}(G)$, then $G_{A}$ is a normal subgroup of $G$.

Proof. By Proposition 4.6, $G_{A}$ is a subgroup of $G$. Moreover $G_{A} \neq \emptyset$. Let $x \in G_{A}$ and let $y \in G$. Then

$$
\text { and } A^{U}\left(y x y^{-1}\right)=A^{U}\left((y x) x^{-1}\right)=A^{U}\left(y^{-1}(y x)\right)=A^{U}(x)=A^{U}(e)
$$$$
A^{L}\left(y x y^{-1}\right)=A^{L}\left((y x) x^{-1}\right)=A^{L}\left(y^{-1}(y x)\right)=A^{L}(x)=A^{L}(e)
$$

Thus $y x y^{-1} \in G_{A}$. Hence $G_{A}$ is a normal subgroup of $G$.

It is clear that if $A$ is a (usual) normal subgroup of $G$, then $A=$ $\left[\chi_{A}, \chi_{A}\right] \in \operatorname{IVNG}(G)$ and $G_{A}=A$. 
Definition 5.5. Let $A \in \operatorname{IVNG}(G)$. Then the quotient group $G / G_{A}$ is called the interval-valued fuzzy quotient subgroup (in short, $I V Q G$ ) of $X$ with respect to $A$.

Now let $\pi: G \rightarrow G / G_{A}$ be the natural projection.

Proposition 5.6. If $A \in \operatorname{IVNG}(G)$ and $B \in D(I)^{G}$, then $\pi^{-1}(\pi(B))=$ $G_{A} \circ B$.

Proof. Let $x \in G$. then

$$
\begin{aligned}
& \pi^{-1}(\pi(B))^{L}=\pi(b)^{L}(\pi(x)) \\
& =\bigvee_{\pi(y)=\pi(x)} B^{L}(y)=\bigvee_{x y^{-1} \in G_{A}} B^{L}(y)
\end{aligned}
$$

and

$$
\begin{aligned}
& \pi^{-1}(\pi(B))^{U}=\pi(b)^{U}(\pi(x)) \\
& =\bigvee_{\pi(y)=\pi(x)} B^{U}(y)=\bigvee_{x y^{-1} \in G_{A}} B^{U}(y) .
\end{aligned}
$$

On the other hand

$$
\left(G_{A} \circ B\right)^{L}(x)=\bigvee_{x y=x}\left[G_{A}(z) \wedge B^{L}(y)\right]=\bigvee_{z=x y^{-1} \in G_{A}} B^{L}(y)
$$

and

$$
\left(G_{A} \circ B\right)^{U}(x)=\bigvee_{x y=x}\left[G_{A}(z) \wedge B^{U}(y)\right]=\bigvee_{z=x y^{-1} \in G_{A}} B^{U}(y)
$$

Thus $\pi^{-1}(\pi(b))(x)=\left(G_{A} \circ B\right)(x)$ for each $x \in G$. Hence $\pi^{-1}(\pi(B))=$ $G_{A} \circ B$.

\section{Interval-valued fuzzy subrings and ideals}

Definition 6.1. Let $(R,+, \cdot)$ be a ring and let $\tilde{0} \neq A \in D(I)^{R}$. Then $A$ is called an interval-valued fuzzy subring (in short, $I V R$ ) in $R$ if it satisfies the following conitions:

(i) $A$ is an IVG in $R$ with respect to the operation " +" (in the sense of Definition 4.1).

(ii) $A$ is an IVGP in $R$ with respect to the operation "." (in the sense of Definition 3.4 or Definition $3.4^{\prime}$ ).

We will denote the set of all IVRs of $R$ as $\operatorname{IVR}(\mathrm{R})$. 
Example 6.1. Consider the ring $\left(\mathbb{Z}_{2},+, \cdot\right)$, where $\mathbb{Z}_{2}=\{0,2\}$. We define the mapping $A: \mathbb{Z}_{2} \rightarrow D(I)$ as follows: $A(0)=[0.2,0.7]$ and $A(1)=[0.5,0.6]$. Then we can see that $A \in \operatorname{IVR}\left(\mathbb{Z}_{2}\right)$.

Remark 6.1. (1)If $A$ is a fuzzy subring of a $\operatorname{ring} R$, then $[A, A] \in \operatorname{IVR}(R)$

(2)If $A \in \operatorname{IVR}(R)$, then $A^{L}$ and $A^{U}$ are fuzzy subrings of $R$.

The following is the immediate result of Definition $3.4^{\prime}$ and Result 4.B.

Proposition 6.2. Let $R$ be a ring and let $\tilde{0} \neq A \in D(I)^{R}$. Then $A \in$ $\operatorname{IVR}(R)$ if and only of for any $x, y \in R$,

(i) $A^{L}(x-y) \geq A^{L}(x) \wedge A^{L}(y)$ and $A^{U}(x-y) \geq A^{U}(x) \wedge A^{U}(y)$.

(ii) $A^{L}(x y) \geq A^{L}(x) \wedge A^{L}(y)$ and $A^{U}(x y) \geq A^{U}(x) \wedge A^{U}(y)$.

The following is easily seen.

Proposition 6.3. Let $R$ be a ring. Then $A$ is a subring of $R$ if and only if $\left[\chi_{A}, \chi_{A}\right] \in \operatorname{IVR}(R)$.

Definition 6.4. Let $R$ be a ring and let $\tilde{0} \neq A \in \operatorname{IVR}(R)$. Then $A$ is called an:

(1) interval-valued fuzzy left ideal (in short, $I V L I)$ in $R$ if $A^{L}(x y) \geq$ $A^{L}(y)$ and $A^{U}(x y) \geq A^{U}(y)$ for any $x, y \in R$.

(2) interval-valued fuzzy right ideal (in short, $I V R I)$ in $X$ if $A^{L}(x y) \geq$ $A^{L}(x)$ and $A^{U}(x y) \geq A^{U}(x)$ for any $x, y \in R$.

(3) interval-valued fuzzy ideal (in short, $I F I$ ) in $X$ if it both an IVLI and an IVRI in $R$.

We will denote the set of all IVLIs [resp. IVRIs and IVIs] of a ring $R$ as $\operatorname{IVLI}(R)[$ resp. $\operatorname{IVRI}(R)$ and $\operatorname{IVI}(R)]$.

Example 6.4. Consider the ring $\left(\mathbb{Z}_{4},+, \cdot\right)$, where $\mathbb{Z}_{4}=\{0,1,2,3\}$. We define the mapping $A: \mathbb{Z}_{4} \rightarrow D(I)$ as follows: $A(0)=[0.2,0.8], A(1)=$ $[0.3,0.6]=A(3)$, and $A(2)=[0.4,0.5]$. Then we can easily see that $A \in$ $\operatorname{IVI}\left(\mathbb{Z}_{4}\right)$

Remark 6.4. (1) If $A$ is a fuzzy [resp. left, right] ideal of a ring $R$, then $\left[A, A^{c}\right] \in \operatorname{IVI}(R)[\operatorname{resp} . \operatorname{IVLI}(R)$ and $\operatorname{IVRI}(R)]$. 
(2) If $A \in \operatorname{IVI}(R)[$ resp. $\operatorname{IVLI}(R)$ and $\operatorname{IVRI}(R)]$, then $A^{L}$ and $A^{U}$ are fuzzy [resp. left and right] ideals of $R$.

The following can be directly verified.

Proposition 6.5. Let $R$ be a ring and let $\tilde{0} \neq A \in D(I)^{R}$. Then $A$ is an IVI[resp. IFLI and IFRI] of $R$ if and only of for any $x, y \in R$,

(i) $A^{L}(x-y) \geq A^{L}(x) \wedge A^{L}(y)$ and $A^{U}(x-y) \geq A^{U}(x) \vee A^{U}(y)$.

(ii) $A^{L}(x y) \geq A^{L}(x) \vee A^{L}(y)$ and $A^{U}(x y) \geq A^{U}(x) \vee A^{U}(y)$ [resp. $A^{L}(x y) \geq A^{L}(y)$ and $A^{U}(x y) \geq A^{U}(y), A^{L}(x y) \geq A^{L}(x)$ and $A^{U}(x y) \geq$ $\left.A^{U}(x)\right]$.

The following is easily seen.

Proposition 6.6. Let $R$ be a ring. Then $A$ is an ideal [resp. a left ideal and a right ideal] of $R$ if and only if $\left[\chi_{A}, \chi_{A}\right] \in \operatorname{IVI}(R)[\operatorname{resp} \operatorname{IVLI}(R)$ and $\operatorname{IVRI}(R)]$.

Proposition 6.7. Let $R$ be a skew field (also division ring) and let $\tilde{0} \neq A \in D(I)^{R}$. Then $A$ is an IFI(IFLI, IFRI) of $R$ if and only if $A^{L}(x)=A^{L}(e) \leq A^{L}(0)$ and $A^{U}(x)=A^{U}(e) \geq A^{U}(0)$ for any $0 \neq x \in R$, where 0 is the identity of $R$ for " + " and $e$ is the identity of $R$ for ".".

Proof. $(\Rightarrow)$ : Suppose $A \in \operatorname{IVLI}(R)$ and let $0 \neq x \in R$. Then

$$
A^{L}(x)=A^{L}(x e) \geq A^{L}(e), A^{L}(e)=A^{L}\left(x^{-1} x\right) \geq A^{L}(x)
$$

and

$$
A^{U}(x)=A^{U}(x e) \geq A^{U}(e), A^{U}(e)=A^{U}\left(x^{-1} x\right) \geq A^{U}(x) .
$$

Thus $A(x)=A(e)$. On the other hand,

$$
\text { and } A^{U}(0)=A^{U}(e-e) \geq A^{U}(e) \wedge A^{U}(e)=A^{U}(e) .
$$$$
A^{L}(0)=A^{L}(e-e) \geq A^{L}(e) \wedge A^{L}(e)=A^{L}(e)
$$

So $A^{L}(e) \leq A^{L}(0)$ and $A^{U}(e) \leq A^{U}(0)$. Hence the necessary conditions hold.

$(\Leftarrow)$ : Suppose the necessary conditions hold. Let $x \in R$. Then we have four cases:

(i) $x \neq 0, y \neq 0$ and $x \neq y$ (ii) $x \neq 0, y \neq 0$ and $x=y$

(iii) $x \neq 0, y=0$ (iv) $x=0, y \neq 0$. 
Case (i) Suppose $x \neq 0, y \neq 0$ and $x \neq y$. Then

$$
\begin{aligned}
& A^{L}(x-y)=A^{L}(e) \geq A^{L}(x) \wedge A^{L}(y), \\
& A^{U}(x-y)=A^{U}(e) \geq A^{U}(x) \wedge A^{U}(y)
\end{aligned}
$$

and

$A^{L}(x y)=A^{L}(e) \geq A^{L}(x) \vee A^{L}(y)$,

$A^{U}(x y)=A^{U}(e) \geq A^{U}(x) \vee A^{U}(y)$.

Case(ii): Suppose $x \neq 0, y \neq 0$ and $x=y$. Then

$$
A^{L}(x-y)=A^{L}(0) \geq A^{L}(x) \wedge A^{L}(y),
$$$$
A^{U}(x-y)=A^{U}(0) \geq A^{U}(x) \wedge A^{U}(y)
$$

and

$$
A^{L}(x y)=A^{L}(e) \geq A^{L}(x) \vee A^{L}(y),
$$$$
A^{U}(x y)=A^{U}(e) \geq A^{U}(x) \vee A^{U}(y) \text {. }
$$

Case(iii): Suppose $x \neq 0$ and $y=0$. Then

$$
\begin{aligned}
& A^{L}(x-y)=A^{L}(x)=A^{L}(e) \geq A^{L}(x) \wedge A^{L}(y), \\
& A^{U}(x-y)=A^{U}(x)=A^{U}(0) \geq A^{U}(x) \wedge A^{U}(y)
\end{aligned}
$$

and

$A^{L}(x y)=A^{L}(0) \geq A^{L}(x) \vee A^{L}(y)$,

$A^{U}(x y)=A^{U}(0) \geq A^{U}(x) \vee A^{U}(y)$.

Case(iv): It is similar to case(iii).

In all, $A \in \operatorname{IVI}(R)$. This completes the proof.

Remark 6.8. Proposition 6.5 shows that an IVLI(IVRI) is an IVI in a skew field.

The following gives a characteristic of a (usual) field by an IVI.

Proposition 6.9. Let $R$ be a commutative ring with a unity $e$. If for $A \in \operatorname{IVI}(R), A^{L}(x)=A^{L}(e) \leq A^{L}(0)$ and $A^{U}(x)=A^{U}(e) \leq A^{U}(0)$ for each $0 \neq x \in R$, then $R$ is a field.

Proof. Let $A$ be an ideal of $R$ such that $A \neq R$. Then clearly $A=$ $\left[\chi_{A}, \chi_{A}\right] \in \operatorname{IVI}(R)$ such that $A \neq \tilde{1}$. Thus there exists $y \in R$ such that $y \notin A$. Thus $\chi_{A}(y)=0$. By the hypothesis, $\chi_{A}(x)=\chi_{A}(e) \leq \chi_{A}(0)$, for each $0 \neq x \in X$. So $\chi_{A}(0)=1$, i.e., $A=\{0\}$. Hence $R$ is a field.

\section{References}

[1] K.Atanassov, Intuitionistic fuzzy sets, Fuzzy Sets and Systems,20(1986) $87-96$.

[2] K.Atanassov and G.Gargov, Interval-valued intuitionistic fuzzy sets, Fuzzy sets and Systems 31(1989) $343 \sim 349$. 
[3] Baldev Benerjee and Dhiren Kr.Basnet, Intuitionistic fuzzy subrings and ideals, J.Fuzzy Math 11(1)(2003) 139-155.

[4] R. Biswas, Rosenfeld's fuzzy subgroups with interval-valued membership functions, Fuzzy set and systems 63(1995) 87-90.

[5] D.Çoker, An introduction to intuitionistic fuzzy topological spaces, Fuzzy Sets and Systems 88(1997) 81-89.

[6] D.Çoker and A.Haydar Es, On fuzzy compactness in intuitionstic fuzzy topological spaces, J.Fuzzy Math. 3(1995) 899-909.

[7] M.B.Gorzalczany, A method of inference in approximate reasoning based on interval-values fuzzy, sets, Fuzzy sets and Systems 21(1987) $1-17$.

[8] K.Hur, S.Y.Jang and H.W.Kang, Intuitionistic fuzzy subgroupoids, International Journal of Fuzzy Logic and Intelligent Systems 2(1)(2002) 92-147.

[9] K.Hur, H.W.Kang and H.K.Song, Intuitionistic fuzzy subgroups and subrings. Honam Math.J. 25(1)(2003) 19-41.

[10] K.Hur, J.G.Lee and J.Y.Choi, Interval-valued fuzzy relations, J. Korean Institute of Intelligent systems 19(3)(2009) 425-432.

[11] W.J.Liu Fuzzy invaiant subgroups and fuzzy ileds, Fuzzy sets and Systems 8(1982) 133189.

[12] T.K.mondal and S.K.Samanta, Topology of interval-valued fuzzy sets, Indian J. Pure Appl. Math. 30(1)(1999) 20-38.

[13] L.A.Zadeh, Fuzzy sets, Inform and Control 8(1965) 338-353.

[14] _, The concept of a linguistic variable and its application to approximate reasoning-I, Inform. Sci 8(1975) 199-249.

*Dept of Mathematics Education,

Woosuk University,

Hujong-Ri, Samrae-Eup,

Wanju-Kun, cheonbuk, Korea 560-701

E-mail: khwon@woosuk.ac.kr

** Division of Mathematics and Informational Statistics, and Nanoscale Science and Technology Institute, Wonkwang University,

Iksan, chonbuk, Korea 570-749

E-mail: kulhur@wonkwang.ac.kr 